\title{
Operative Versus Nonoperative Treatment After Acute Patellar Dislocation: Which Is More Effective at Reducing Recurrence in Adolescents?
}

\author{
Julie A. Fuller, Heidi L. Hammil, Kelly J. Pronschinske, and Chris J. Durall
}

\begin{abstract}
Clinical Scenario: Acute patellar dislocations during adolescence often lead to future patellar instability. Two common treatment options include nonoperative treatment or operative repair of injured structures. Focused Clinical Question: In adolescents with acute patellar dislocation, how does operative stabilization compare with nonoperative treatment for reducing dislocation recurrence? Summary of Key Findings: Three studies were included: 2 randomized controlled trials and 1 nonrandomized study. All studies compared operative and nonoperative treatment outcomes in adolescents who experienced an acute patellar dislocation. Each study included nonoperative treatment such as patellar bracing and quadriceps strengthening. The operative treatments utilized in each study included lateral retinacular release and medial retinacular repair. All 3 of the studies included a follow-up of at least 6 years. Two of the studies concluded there to be no significant difference between treatment groups regarding redislocation rate, pain, and function. The third study reported a lower redislocation rate following operative treatment. Clinical Bottom Line: Reviewed evidence suggests that outcomes are similar when comparing operative and nonoperative treatment approaches with little agreement as to which is the optimal plan of action. Strength of Recommendation: One level II randomized controlled trial and a level III nonrandomized study suggest that patellar dislocation recurrence rates are similar among operative and nonoperative treatment approaches, while another level II randomized controlled trial suggests that an operative approach is superior.
\end{abstract}

Keywords: patellar instability, patellar stabilization, quadriceps exercise, patellar bracing

\section{Clinical Scenario}

Acute patellar dislocation most commonly affects young, active individuals. ${ }^{1}$ Regardless of treatment, patellar dislocation may lead to patellofemoral instability. Two common approaches for treating acute patellar dislocation are surgical stabilization and nonoperative treatment by patellar bracing and exercise. ${ }^{2}$ This review was conducted to determine if current best evidence supports one of these treatment approaches over the other for reducing dislocation recurrence in adolescents.

\section{Focused Clinical Question}

In adolescents with acute patellar dislocation, how does operative stabilization compare with nonoperative treatment for reducing dislocation recurrence?

\section{Summary of Search, "Best Evidence" Appraised, and Key Findings}

- The literature was searched for studies that compared outcomes following surgical stabilization or nonoperative treatment of acute patellar dislocation in adolescents.

- The search yielded 2 level II randomized controlled trials 2,3 and 1 level III nonrandomized study ${ }^{1}$ that directly compared

The authors are with the University of Wisconsin-La Crosse, La Crosse, WI, USA. Durall is also with the Student Health Center, Physical Therapy Unit, University of Wisconsin-La Crosse, La Crosse, WI, USA. Durall (cdurall@uwlax.edu) is corresponding author. patellar redislocation rate, knee function, and patellofemoral pain between the 2 treatment approaches.

- In 2 of the 3 reviewed studies, the authors reported no difference in dislocation recurrence rates with operative or nonoperative treatment. ${ }^{1,2}$ In the third study, recurrence rates were reportedly lower in the operative treatment group, although, in contrast to the other studies, most of this surgical group received an additional bony realignment procedure. ${ }^{3}$ The 3 reviewed studies all reported no difference between groups utilizing unique functional outcome measures. ${ }^{1-3}$ Two of the reviewed studies also reported no difference in pain levels based on treatment approach, ${ }^{1,2}$ while the third study did not include an outcome measure related to pain. ${ }^{3}$

\section{Clinical Bottom Line}

Reviewed evidence suggests that patellar redislocation rate, knee function, and patellofemoral pain intensity are all similar with operative or nonoperative treatment of acute patellar dislocation in adolescents. There was no consensus in the reviewed studies regarding the optimal nonoperative strategy to treat acute patellar dislocations, although quadriceps strengthening and bracing were utilized in all 3 reports.

\section{Strength of Recommendation}

Studies to date on this topic are limited in number, moderate in quality (levels II and III), and somewhat inconsistent in their findings. Thus, our conclusion that patellar redislocation rates with operative and nonoperative management are similar is based on a moderate degree of confidence. 


\section{Search Strategy}

\section{Terms Used to Guide Search Strategy}

- Patient/Client Group: instability, unstable, hypermobile, dislocation and knee, patella, patellofemoral AND acute, first, initial, primary and adolescents, young adults, and children

- Intervention: physiotherapy, rehabilitation, physical therapy, conservative treatment, nonoperative treatment, and nonsurgical treatment

- Comparison: surge, surgery, operative treatment, repair, and arthroscopy

- Outcome(s): patellar dislocation, redislocation, redislocation, and recurrence rate

\section{Sources of Evidence Searched}

- PubMed

- CINAHL Plus

- SPORTDiscus

- MEDLINE

- Alt HealthWatch

- Health Professions Database by EBSCOhost

- Cochrane Database of Systematic Reviews

- PsycINFO

- Additional resources obtained by manual search of reference lists

\section{Inclusion and Exclusion Criteria}

\section{Inclusion}

- Confirmed diagnosis of patellar dislocation based on ability to dislocate patella under anesthesia, presence of locked dislocation or reduced dislocation within previous 1 to 2 weeks

- Studies that compared operative to nonoperative treatment after acute patellar dislocation

- Studies that included participants 8-16 years old

- Limited to humans

- Limited to English language

- Limited to the last 10 years (2006-2016)

\section{Exclusion}

- History of surgery or serious injury on affected knee or leg

- Studies only comparing different operative treatments for acute patellar dislocation

\section{Results of Search}

An extensive search of the literature yielded 42 articles; however, only 3 studies $^{1-3}$ met the eligibility requirements and were considered for review. These studies are listed in Table 1 and summarized in Table 2. All 3 studies examined the effect of operative versus nonoperative treatment after acute patellar dislocation in individuals between the ages of 8 and16.
Table 1 Summary of Study Designs of Retrieved Articles

\begin{tabular}{llcl}
\hline $\begin{array}{l}\text { Level of } \\
\text { evidence }\end{array}$ & $\begin{array}{l}\text { Study design/ } \\
\text { Methodology }\end{array}$ & $\begin{array}{l}\text { Number } \\
\text { located }\end{array}$ & Author \\
\hline II & $\begin{array}{l}\text { Randomized } \\
\text { controlled trial }\end{array}$ & 2 & $\begin{array}{l}\text { Palmu et } \mathrm{al}^{2} \\
\text { Regalado et } \mathrm{al}^{3}\end{array}$ \\
III & $\begin{array}{l}\text { Nonrandomized } \\
\text { controlled trial }\end{array}$ & 1 & \begin{tabular}{l} 
Apostolovic $_{\text {et al }}{ }^{1}$ \\
\hline
\end{tabular} \\
\hline
\end{tabular}

\section{Best Evidence}

The studies identified in Table 1 are the best available evidence found for this review. Two of the included articles are level II evidence, and the third article is level III evidence. Level of evidence was determined based on the 2011 criteria from the Centre for Evidence-Based Medicine.

\section{Implications for Practice, Education, and Future Research}

Different strategies have been recommended in the treatment of acute patellar dislocation including bracing, strengthening, and operative stabilization. ${ }^{4}$ Two of the articles retrieved for this review found no significant differences in patellar redislocation rate, knee function, or patellofemoral pain between operative stabilization and nonoperative treatment with bracing and exercise. ${ }^{1,2}$ In the third study, Regalado et $\mathrm{al}^{3}$ reported a lower redislocation rate after surgery at the 3-and 6-year follow-ups (Figure 1). At 6 years, patients in the operative group had a redislocation rate of $33 \%$ $(5 / 15)$ versus $73 \%$ (11/15) for the nonoperative group. Palmu et $\mathrm{al}^{2}$ reported a similar dislocation recurrence rate of $71 \%$ (20/28) for the nonoperative group at the 14-year follow-up, but the recurrence rate in the operative group was 67\% (24/36). In stark contrast to those studies, Apostolovic et $\mathrm{al}^{1}$ found redislocation rates of $7 \%$ $(1 / 23)$ for the nonoperative group and $9 \%$ (2/14) for the operative group. It is worth noting that the 1 study $^{3}$ favoring operative over nonoperative management had a PEDro score of 3/10, suggesting potential bias and/or methodological shortcomings. Apostolovic et $\mathrm{al}^{1}$ and Palmu et $\mathrm{al}^{2}$ had PEDro scores of 4/10 and 6/10, respectively.

The surgical techniques employed in the 3 studies differed somewhat, making direct comparisons difficult. Lateral retinacular release and medial retinaculum repair were used in all 3 reports. Palmu et $\mathrm{al}^{2}$ also reconstructed/repaired the medial patellotibial and/or patellofemoral ligaments in some cases. In contrast, Regalado et $\mathrm{al}^{3}$ used the modified Roux-Goldthwait procedure, which involves a proximal and distal realignment along with lateral retinacular release and medial retinacular repair. Thus, the Regalado et $\mathrm{al}^{3}$ study was the only one that used a bony realignment procedure (ie, distal realignment) as part of the surgical intervention for the majority (13/16) of patients. This is noteworthy since Regalado et al were the only authors in this review who reported a lower dislocation rate following surgery. Given the disparity in surgical outcomes between the reviewed studies, there is a need for additional investigations comparing the various operative techniques.

Rates of reoperation differed between the studies. At the 6-year follow-up, Regalado et $\mathrm{al}^{3}$ performed additional patellar 
Table 2 Characteristics of Included Studies

\begin{tabular}{|c|c|c|c|}
\hline & Palmu et $\mathrm{al}^{2}$ & Apostolovic et al $^{1}$ & Regalado et al $^{3}$ \\
\hline Study design & $\mathrm{RCT}$ & NRS & $\mathrm{RCT}$ \\
\hline $\begin{array}{l}\text { Level of } \\
\text { evidence }\end{array}$ & II & III & II \\
\hline PEDro & $6 / 10$ & $4 / 10$ & $3 / 10$ \\
\hline
\end{tabular}

Participants 62 patients $(M=16, F=46)$ with a mean age of 13 y (range 9-15), clinically diagnosed with acute patellar dislocation. Randomly assigned into nonoperative group (28 knees) or operative group (36 knees). No significant differences between the groups before intervention.

Intervention(s) Nonoperative treatment by bracing and investigated exercise. For both groups, "thigh muscle exercises" and FWB were started as soon as tolerated. All patients were encouraged to wear orthosis during athletic activities for first 6 mo after injury.

Comparison

The operative group had surgery within $2 \mathrm{wk}$ including direct repair of the damaged medial structures with absorbable suture placement. A lateral release was also performed in all except 4 knees. If patella was dislocatable under anesthesia patient wore removable, knee extension orthosis for $3 \mathrm{wk}$ followed by patella-stabilizing orthosis for 3 wk. If patella was not dislocatable, under anesthesia patient wore patella-stabilizing orthosis for $6 \mathrm{wk}$

Outcome Redislocation rate, function (Kujala score), measure(s) pain (Hughston Visual Analog Scale), and Tegner activity scores were assessed at baseline for 2, 6, and $14 \mathrm{y}$.

Main findings Redislocation rate was similar in the nonoperative group $(20 / 28 ; 71 \%)$ and in the operative group $(24 / 36 ; 67 \%)$. A total of $52 \%$ of the recurrent dislocations occurred within 2 y after primary injury. Kujala (14-y follow-up) mean scores operative $=83$ and nonoperative $=84$ $(P=.49$; effect size $=0.055)$. Kujala $(P=.06)$ and Tegner activity scores $(P=.01)$ improved more for males than females. At 14-y follow-up, subjective result was good-excellent for $75 \%$ of nonoperative group and $66 \%$ of operative group (nonsignificant difference at $P=.48$; relative risk $=1.14)$.

Conclusion No significant or clinical between-group differences in redislocation rate, function, pain, or Tegner activity score. Operative approach did not improve long-term outcomes. Investigators did not advocate routine repair of the damaged medial structures for acute patellar dislocation in adolescents.
37 patients $(M=9, F=28)$ with a mean age of 13.8 y (range 12-16), clinically diagnosed with primary acute patellar dislocation. Fourteen patients with an intraarticular loose body $>8 \mathrm{~mm}$ were assigned to the operative group. Other differences between groups before intervention were not reported.

Nonoperative treatment over a mean of 3 wk including: closed reduction of the patella, immobilization, cryotherapy within the first day of injury, aspiration of effusion, and isometric quadriceps exercises as pain levels decreased. Both groups underwent aspiration of effusion.

The operative group had surgery within $10 \mathrm{~d}$ of initial injury including hemostasis, and loose-body removal. Other surgical procedures were performed based on extent of individual injury including extent of injury to medial structures, mediolateral patellar mobility, patellofemoral alignment, and other factors influencing patellar stability.

Redislocation rate, function (CKRS) and pain were assessed at initial treatment and rehabilitation, typically $8-10 \mathrm{mo}$, and annual follow-ups were performed for 5-8 y.

There was no significant difference in redislocation rate between the nonoperative group $(1 / 23 ; 9 \%)$ and the operative group $(2 / 14 ; 7 \%)$. There were 29 successful outcomes (20 nonoperative and 9 operative; relative risk $=1.35$ ). There was no significant or clinical difference in successful outcomes between the groups as measured by CKRS $(P=.09$; effect size $=$ $0.13)$.

No statistical or clinical between-group differences in redislocation rate, function, or pain. Investigators advocated nonoperative treatment due to equivalent results yet lower risk compared with surgery.
36 patients $(\mathrm{M}=14, \mathrm{~F}=22)$ with a mean age of 13.5 y (range 8-16), clinically diagnosed with acute primary patellar dislocation. Randomly assigned to nonoperative treatment (nonoperative group; $\mathrm{n}=20$ ) or operative treatment (operative group; $\mathrm{n}=16$ ). No significant differences between the groups before intervention.

Nonoperative treatment by bracing and exercise. Both groups were put in lateral patellar support brace with $30^{\circ}$ flexion for $3 \mathrm{wk}$ and then $90^{\circ}$ flexion for next $3 \mathrm{wk}$. Brace removed after $6 \mathrm{wk}$, and patients were instructed to complete full ROM. FWB from the start. Rehab protocol (ROM as tolerated, isometric quadriceps strengthening, progressed to isokinetic strengthening when knee ROM was pain free) was the same for both groups. 1-2 physical therapy visits per month for 6 mo. Operative group had surgery based on degree of patellar instability and tilt (types I-IV). LRR with proximal/distal realignment plus medial imbrication was performed based on type of patellar instability and/or tilt.

Redislocation rate, knee function (excellent/good/poor), and overall satisfaction (satisfied/dissatisfied) were assessed at 3- and 6-y follow-ups.

The patellar redislocation rate at $6 \mathrm{y}$ after the primary procedure was higher in the nonoperative group $(11 / 15 ; 73 \%)$ than in the operative group $(5 / 15 ; 33 \%)(P=.02$; relative risk $=2.2$ ). Knee function was slightly better at the 6-y follow-up in the operative group than in the nonoperative group. Most participants in the nonoperative group $(11 / 15 ; 73 \%)$ and operative group $(13 / 15 ; 87 \%)$ had excellent or good knee function at the 6-y follow-up, but not significant overall $(P>.05)$. Only 6/30 $(20 \%)$ of patients were dissatisfied with their procedure at the 6-y follow-up.

Statistical difference between groups at 3- and 6-y follow-ups in redislocation rate. No statistical or clinical between-group differences in function or overall satisfaction. Both modes of treatment are feasible in adolescents; however, increased rates of redislocation are higher with a nonoperative approach.

Abbreviations: CKRS, Cincinnati knee rating system; F, female; FWB, full weightbearing; LRR, lateral retinacular release; M, male; NRS, nonrandomized study; RCT, randomized controlled trial; ROM, range of motion. 


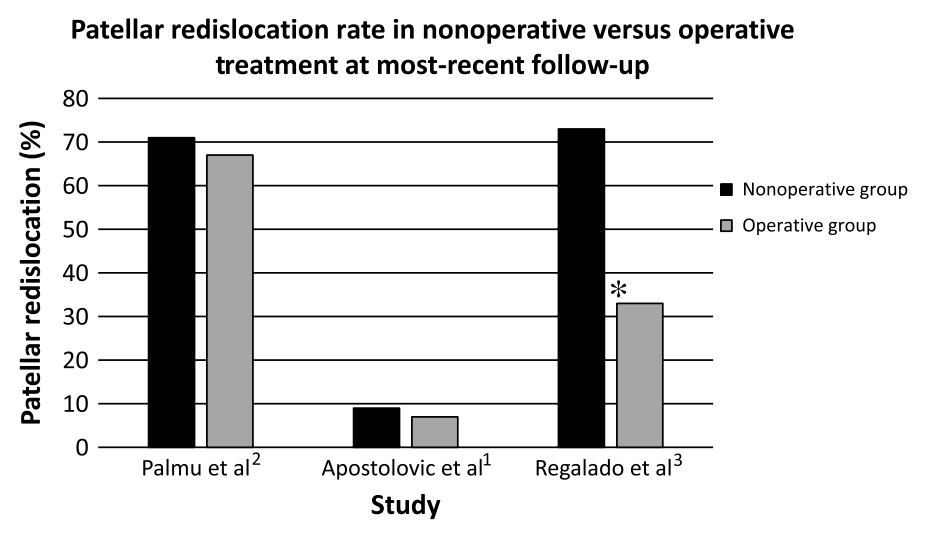

Figure 1 - Patellar redislocation rates from the reviewed studies. *Statistical significance.

stabilization procedures on 0 of 15 patients $(0 \%)$ in the operative group and 4 of $15(27 \%)$ in the nonoperative group. Palmu et $\mathrm{al}^{2}$ reported reoperations at the 3-year follow-up of 44\% (16/36) in the operative group and 39\% (11/28) in the nonoperative group. Most of the first redislocations in this study occurred within 2 years after the primary dislocation, and most patients had more than 1 redislocation. Apostolovic et $\mathrm{al}^{1}$ performed reoperations on 4 of 14 patients $(29 \%)$ in the operative group and 4 of $23(17 \%)$ in the nonoperative group. Considering all follow-ups, the percentage of patients in the nonoperative groups who went on to have patellar stabilization surgery was $17 \%$ in the Apostolovic et al ${ }^{1}$ study, $39 \%$ in the Palmu et $\mathrm{al}^{2}$ study, and $45 \%$ in the Regalado et $\mathrm{al}^{3}$ study for an overall average of $34 \%$.

Nonoperative treatment differed somewhat between the reviewed studies, although all of the authors used exercise and bracing. ${ }^{1-3}$ Apostolovic et $\mathrm{al}^{1}$ also used cold packs during the first 24 hours after injury, immobilization, and later aspiration of the knee effusion. Palmu et $\mathrm{al}^{2}$ employed a period of immobilization followed by "thigh muscle exercises." Apostolovic et $\mathrm{al}^{1}$ and Regalado et al $^{3}$ utilized "isometric quadriceps exercises." Unfortunately, none of the 3 studies provided descriptions of the specific exercises or exercise parameters (sets, repetitions, and frequency). Thus, we are unable to provide detailed recommendations based on these studies, although symptom-limited quadriceps strengthening exercises seem sensible. The authors of a 2010 systematic review on rehabilitation following lateral patellar dislocation concluded that there was little evidence to support 1 particular exercise approach for preventing future dislocations. The most common interventions used in that review included immobilization and isometric and isotonic quadriceps strengthening exercises such as straight leg raises, squats, and lunges. ${ }^{4}$

As with exercise, patellar stabilizing bracing was used in all the studies we reviewed although details were somewhat sparse. Apostolovic et $\mathrm{al}^{1}$ indicated that nonoperative treatment included "immobilization" but did not provide further information. Patients in the Regalado et $\mathrm{al}^{3}$ study used a "conventional lateral patellar support-Rehab brace" that permitted $30^{\circ}$ flexion for 3 weeks and $90^{\circ}$ flexion for the next 3 weeks. In the Palmu et $\mathrm{al}^{2}$ study, patients in both groups who had a dislocatable patella under anesthesia were managed with a removable knee extension orthosis (Immobilizer; Camp International, Jackson, MI) for 3 weeks, followed by a patella-stabilizing orthosis (Patella Stabilizer; Camp International) for an additional 3 weeks. Patients with a nondislocatable patella under anesthesia used a patella-stabilizing orthosis for 6 weeks. Given the inconsistency with bracing in these studies we are not able to provide specific bracing recommendations.

Another study ${ }^{5}$ comparing operative or nonoperative management for patellar dislocation was examined for this review, although the mean age of patients was 20 years, and so the study was not included. Interestingly, those authors also found that surgical stabilization neither reduce rates of redislocation ( $16.7 \%$ vs $20 \%$ for nonoperative group) nor it produce better subjective functional outcomes. Likewise, Nikku et $\mathrm{al}^{6}$ reported redislocation rates at the 7-year follow-up of $31 \%$ and $39 \%$ for the operative and nonoperative groups, respectively in a group with a mean age of 20. Thus, it appears that surgical and nonsurgical management of patellar dislocation tends to yield similar results in the skeletally mature and adolescents.

Individual characteristics and goals should be taken into consideration when choosing an acute patellar dislocation treatment approach. Unless future studies show a more definitive benefit for surgical management, exercise and bracing should be considered initially. Exercise and bracing are less invasive and likely to be less expensive than surgery. Future investigators should provide specific details to allow for both study replication and direct comparisons of different braces and exercise protocols.

\section{References}

1. Apostolovic M, Vukomanovic B, Slavkovic N, et al. Acute patellar dislocation in adolescents: operative versus nonoperative treatment. Int Orthop. 2011;35(10):1483-1487. PubMed ID: 21574051 doi:10. 1007/s00264-011-1265-Z

2. Palmu S, Kallio P, Donell S, Helenius I, Nietosvaara Y. Acute patellar dislocation in children and adolescents: a randomized clinical trial. J Bone Joint Surg. 2008;90(3):463-470. PubMed ID: 18310694 doi:10.2106/JBJS.G.00072

3. Regalado G, Lintula H, Kokki H, Kroger H, Vaatainen U, Eskelinen M. Six-year outcome after non-surgical versus surgical treatment of acute primary patellar dislocation in adolescents: a prospective randomized trial. Knee Surg Sports Traumatol Arthrosc. 2016;24(1): 6-11. PubMed ID: 25193570 doi:10.1007/s00167-014-3271-3

4. Smith T, Davies L, Chester R, Clark A, Donell S. Clinical outcomes of rehabilitation for patients following lateral patellar dislocation: a systematic review. Physiotherapy. 2010;96(4):269-281. PubMed ID: 21056161 doi:10.1016/j.physio.2010.02.006

5. Christiansen SE, Jakobsen BW, Lund B, Lind M. Isolated repair of the medial patellofemoral ligament in primary dislocation of the patella: a prospective randomized study. Arthroscopy. 2008;24(8): 881-887. PubMed ID: 18657736 doi:10.1016/j.arthro.2008.03.012

6. Nikku R, Nietosvaara Y, Aalto K, Kallio PE. Operative treatment of primary patellar dislocation does not improve medium term outcome: a 7-year follow-up report and risk analysis of 127 randomized patients. Acta Orthop. 2005;76(5):699-704. PubMed ID: 16263618 doi:10.1080/17453670510041790 